\title{
Continuous Intraoperative Recurrent Laryngeal Nerve Monitoring: Techniques, Applications, and Controversies
}

\author{
Julia I. Staubitz ${ }^{1} \cdot$ Thomas J. Musholt $^{1}$ (i) \\ Accepted: 7 May 2021 / Published online: 7 June 2021 \\ (C) The Author(s) 2021
}

\begin{abstract}
Purpose of Review Purpose of the present review is to illustrate the current state of the art concerning continuous intraoperative recurrent laryngeal nerve monitoring (cIONM) for thyroid surgery.

Recent Findings cIONM potentially leads to an improved postoperative vocal cord palsy rate, compared to the intermittent technique. There are currently two main approaches for cIONM: either conventional cIONM based on vagal nerve stimulation or experimental methods, which do not require the positioning of a vagal nerve electrode. One of these methods is the recently described technique "LAR-cIONM," which utilizes the laryngeal adductor reflex.

Summary cIONM represents an advancement of intermittent nerve monitoring, which allows for an immediate reaction to signal changes. Threshold values and guidelines to prevent recurrent laryngeal nerve palsy were validated for the direct stimulation of the vagal nerve and require verification for alterative cIONM methods, including LAR-cIONM.
\end{abstract}

Keywords Continuous intraoperative nerve monitoring $\cdot$ IONM $\cdot$ Thyroidectomy $\cdot$ Neck surgery

\section{Introduction}

\section{Etiology and Impact of Vocal Cord Palsy in Thyroid Surgery}

In thyroid surgery, the risk of postoperative vocal cord palsy (VCP) caused by intraoperative recurrent laryngeal nerve damage is one of the most important complications. Depending on the expertise of the operating surgeon, transient and permanent vocal cord palsy (VCP) rates resulting from thyroid surgery range from 1 to $18.6 \%$ [1]. Impairment of glottic closure, dysphonia, dysphagia, ineffective cough, and aspiration pneumonia can be the result $[2,3,4 \bullet \bullet]$. When occurring bilaterally, VCP leads to a mechanically based

This article is part of the Topical Collection on Nerve Monitoring in Head and Neck Surgery

Thomas J. Musholt

musholt@uni-mainz.de

Julia I. Staubitz

julia.staubitz@unimedizin-mainz.de

1 Section of Endocrine Surgery, Department of General, Visceral and Transplantation Surgery, University Medical Center Mainz, Johannes Gutenberg-University Mainz, Langenbeckstraße 1, D-55131 Mainz, Germany respiratory insufficiency, which potentially requires tracheotomy (in up to $30 \%$ ) $[2,3,4 \cdot \bullet, 5]$. The prevailing mechanism of injury of the recurrent laryngeal nerve (RLN) during thyroid surgery is traction $[6,7]$. The crucial point, where traction is immediately translated to the RLN, is during preparation at the suspensory ligament of the thyroid gland (Berry's ligament) $[6,7]$. In addition to traction, thermal damage, ligature entrapment, suction-related injury, or even transection can provoke serious and potentially permanent RLN lesions [6, 7]. Depending on the mechanism of injury, segmental (type 1) or global (type 2) RLN loss of signal can occur [4••, 8]. Segmental loss of signal was observed to cause early VCP in $95 \%$ of cases, whereas (less severe) global loss was associated with VCP in $70 \%[6,9 \cdot \bullet]$. Additional risk factors associated with the development of VCP development are autoinflammatory processes (e.g., Graves' disease or Hashimoto's thyroiditis) $[10,11,12 \bullet]$, hospital volume [12-15], previous thyroid surgery of the individual $[14,16]$, and thyroid malignancy $[14,15]$.

\section{Assessment of Vocal Cord Paralysis: Laryngoscopy Is Indispensable}

Laryngoscopy is the gold standard to assess pre- and postoperative vocal cord paralysis and is strongly recommended by the International Neuromonitoring Study Group Guidelines 
(INMSG) and the German association of Endocrine surgeons (CAEK) [2, 8, 17, 18]. Preoperatively documented VCP, which can be detected in 0.5 to $1.5 \%$ of cases, is a relevant indicator for the presence of advanced thyroid malignancies and often associated with the necessity of an extensive operation including potential RLN resection [2]. Yet, routine preoperative laryngoscopy can reveal preexisting VCP also in benign disease, e.g., for compression syndrome caused by large nodular goiter. To only rely on the intraoperative initial stimulation of the vagal nerve may be misleading, since EMG signals can be present despite vocal cord paresis verified by laryngoscopy. Therefore, preoperative laryngoscopy is an essential tool to adequately set up the surgical strategy and to correctly interpret the intraoperative EMG signals. Postoperative laryngoscopy evaluation, on the other hand, is necessary for the objective assessment of the operating surgeon's VCP rate. An important reason why intraoperative nerve monitoring (IONM) cannot replace postoperative laryngoscopy is that an intact RLN function is not completely predictable by a certain electromyogram (EMG) amplitude level (e.g., $100 \mu \mathrm{V}$ threshold) $[19,20]$. Relative changes of the amplitude, especially a drop of more than $50 \%$ of the initially recorded EMG signal, are associated with increased risk of VCP [4••]. Only early postoperative laryngoscopy can determine the vocal cord function within this "grey zone." Similarly, the assessment of patient voice (hoarseness) is not a reliable predictor of vocal cord function, since up to $50 \%$ of patients with VCP can be clinically asymptomatic. Immediate postoperative dysphonia may be concealed by edema and absence of atrophy of the vocal cord muscle, which will occur later in patients with permanent RLN lesions. Therefore, it is important to identify these patients using laryngoscopy, and to refer them to early voice rehabilitation therapy, so that increasing dysphonia in the later course of the disease is avoided.

\section{Intraoperative Vagal Nerve/RLN Monitoring: Conclusions to Be Drawn from the Intermittent Technique}

The identification of the RLN is essential to preserve its functional integrity during surgery $[21,22]$. The use of intermittent IONM was shown to contribute to the intraoperative localization of the RLN $[23,24]$. In this sense, the technique can be used to facilitate safe tissue dissection, as unclear structures can be assessed with the handheld simulation probe before transection [25]. Also rare anatomic variants like an inferior (non-recurrent) laryngeal nerve can be identified by stimulating along the vagal nerve. Yet, the surgeon must be aware that fluid collection around the point of stimulation may impair the flow of electrical current to the nerve and result in failure of stimulation. Bipolar and monopolar probes create characteristic current patterns resulting in a more precise stimulation for bipolar probes, or a more widespread stimulation when monopolar probes are used, which is ideal for IONM mapping in circumscribed areas. Yet, stimulation in close proximity to the larynx, esophagus, or trachea may produce signal artifacts that can only be distinguished from true signals of the vocal muscle by assessment of the EMG curves. In addition, technical problems may occur during the operation as, e.g., disconnection or breaking of wires. Surgeons should always be aware of these potential errors and malfunctions. Therefore, the visual identification of the RLN remains the gold standard. Most important for the determination of the extent of surgery, the intermittent technique allows for the information about a loss of signal after unilateral thyroid resection. For the intermittent technique, a suprathreshold stimulation (1-2 mA) of the vagal nerve and RLN is performed using a handheld stimulation probe, and compound muscle action potentials of the laryngeal muscles are registered via surface electrodes on an endotracheal tube which are positioned at the vocal fold level (Fig. 1A). An optimal initial signal at stimulation of the vagal nerve of $>500 \mu \mathrm{V}$ should be aimed for, to adequately assess a relative signal decrease during the operation [4••]. The systematic assessment of the vagal and the recurrent laryngeal nerve signals before and after unilateral thyroid surgery was strongly recommended by the INMSG [25]. The recommended standard assessment by intermittent IONM is the preliminary stimulation of the vagal nerve ("V1"), followed by preliminary assessment of the ipsilateral RLN ("R1") and the post resection stimulation of the RLN ("R2") and the vagal nerve ("V2") [25, 26]. The stimulation can eventually be complemented by the pre- and post resection assessment of the external branch of the ipsilateral superior laryngeal nerve ("S," responsible of tensing the vocal ligaments via the cricothyroid muscle) resulting in the algorithm "V1-R1-S1R2-V2-S2." After measuring the post resection stimulation results, ipsilateral tissue preparation should be abstained from. The INMSG defined a loss of signal (LOS) as the reduction of the EMG response from an initially satisfying response $>500 \mu \mathrm{V}$ to $<100 \mu \mathrm{V}$ [25]. The intermittent IONM technique further allows for the detection of the point of lesion in case of a LOS [25]. In case of a type 1 (segmental) LOS, stimulation of the vagal nerve and laryngeal recurrent nerve proximal to the point of injury will not lead to an EMG signal, whereas by stimulating the final tract of the recurrent laryngeal nerve, which is distal to the point of injury, an EMG response will be registered. In case of a type 2 (global) LOS, neither distal nor proximal testing of the vagal nerve/RLN axis will lead to an EMG response [8]. The information about a LOS can be used by the operating surgeon to determine the resection extent. In case of benign goiter, a change of the surgical approach from an intended thyroidectomy to lobectomy should be considered, thereby avoiding the potential risk of a bilateral VCP [8]. In case of thyroid malignancy, however, the strategical change to a two-staged procedure must be evaluated carefully, taking into consideration the oncological risk [2, 8]. 

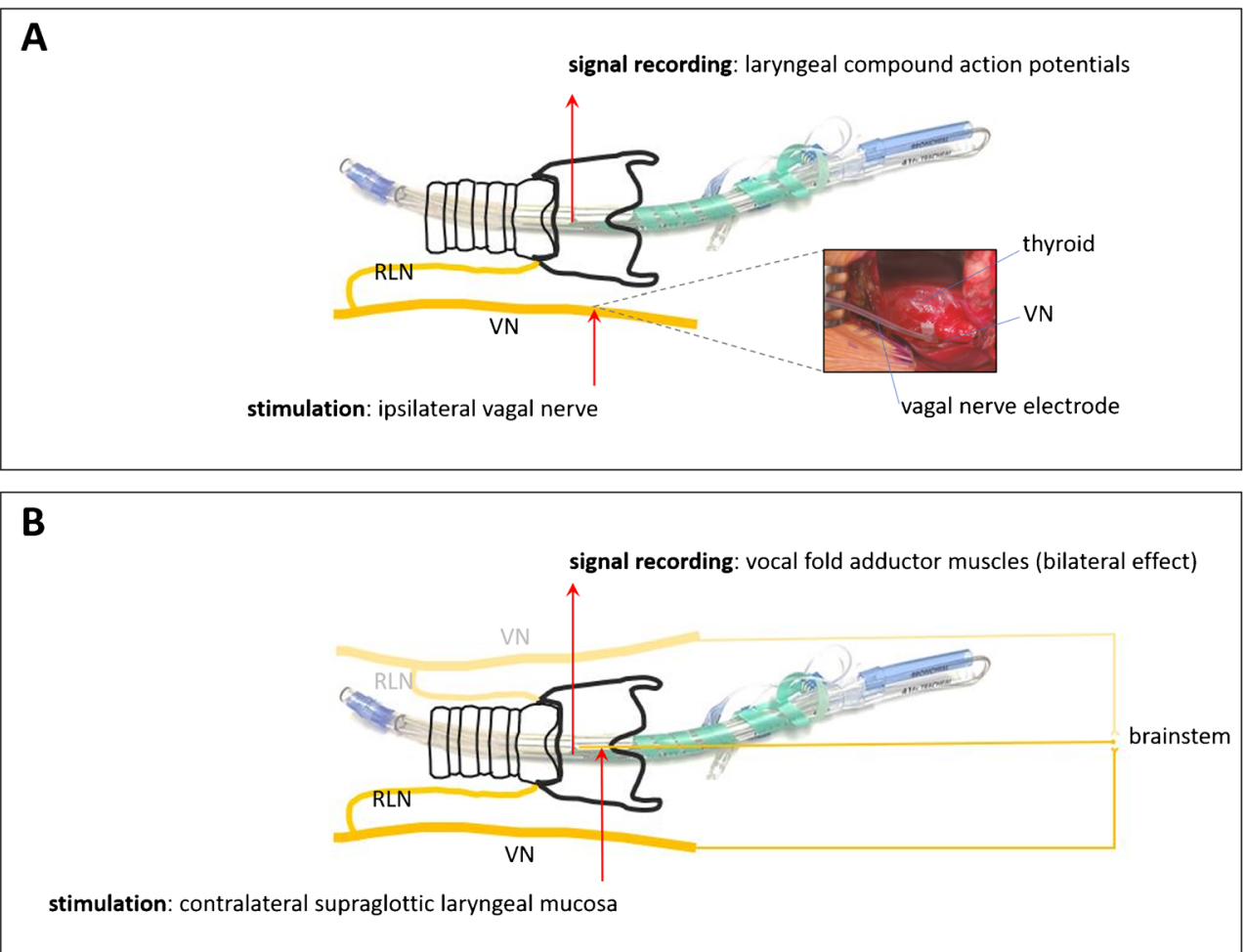

Fig. 1 Scheme of loci for stimulation and signal recording for (A) vagal/ recurrent laryngeal nerve based and (B) laryngeal adductor reflex based continuous intraoperative nerve monitoring; setup for lobectomy of the left thyroid lobe. A Stimulation is performed using a vagal nerve electrode on the side, which is operated on (left thyroid lobe). Compound action potentials of the laryngeal muscles are registered. B Stimulation is performed at the supraglottic mucosa contralateral to the side, which is operated on. The laryngeal adductor reflex elicits bilateral

\section{From Intermittent to Continuous IONM: Real-Time Evaluation of RLN Function During Surgical Maneuvers}

In addition to the advantages of the intermittent technique (which are RLN "mapping” during tissue dissection, assessment of RLN lesion point/type of LOS, and decision guidance towards two-staged thyroidectomy), continuous IONM (cIONM) facilitates a real-time evaluation of the effect of the surgical maneuvers on RLN function [26-28]. There are different approaches to perform cIONM, which primarily differ in the localization of stimulation. The conventional way of cIONM makes use of the vagal nerve/RLN axis: repetitive stimulation is directly exerted on the vagal nerve (Fig. 1A) using different types of vagal nerve electrodes, and resultant vocal fold contractions (compound muscle action potentials) are registered at the vocal fold level, similarly to the intermittent IONM setup [29-31]. In addition, there are alternative techniques, which abstain from a direct stimulation of the vagal nerve. In 2016, transcutaneous intraoperative vagal stimulation was described for cIONM during thyroidectomy and parathyroidectomy [32]. To avoid the placement of a vagal stimulation electrode during endoscopic thyroid surgery, transcutaneous activity of the vocal fold adductors. The reaction from the left side surveys the operative activity and stress on the recurrent laryngeal nerve, whereas the contralateral LAR responses (in unilateral surgeries) could be used to determine, whether any decreased signal that occurs perioperatively is related to nerve stress or merely tube displacement. Currently, endotracheal tubes do not yet allow for simultaneous recording of bilateral LAR responses. VN, vagal nerve; RLN, recurrent laryngeal nerve

vagal nerve stimulation was evaluated and considered not feasible in the experimental setup [33]. Moreover, transcranial motor-evoked potentials of laryngeal muscles were proposed as a feasible alternative for cIONM during thyroid surgery [34]. "LAR-cIONM," a recently described method, which requires endotracheal surface electrodes for both cIONM signal stimulation and -recognition, stands out from these alternative techniques as a very promising example. The technique takes advantage of the laryngeal adductor reflex, a primitive brainstem reflex that protects the tracheobronchial tree from foreign bodies. For LAR-cIONM, the reflex is repetitively elicited by supraglottic mucosal stimulation, and afferent activity runs in the internal superior laryngeal nerve to the brainstem. Efferent activity is transported via the vagal nerve and RLN to laryngeal adductors, and bilateral vocal fold adduction is registered (Fig. 1B) $[35 \cdot, 36,37]$. However, these alternative techniques still hold an experimental status.

\section{Effect of IONM Usage on Postoperative VCP Rate}

Recent European studies were able to demonstrate the beneficial influence of IONM to reduce the risk of transient $[12,38]$ or permanent [13] VCP. Of interest is, that continuous IONM 
techniques were reported to reduce the transient [36] and permanent $[9 \cdot \bullet] \mathrm{VCP}$ rate more effectively than intermittent IONM. These international results contradict contemporary meta-analyses, which concluded that the use of intraoperative nerve monitoring did not contribute to reduced VCP rates [19-21].

\section{Practical Use of IONM in Europe}

A recent analysis of the registry EUROCRINE® showed that European hospitals with focus on endocrine surgery used IONM in over $90 \%$ of operations, which also underwent postoperative laryngoscopy control [12•]. In contrast to previous observations, IONM was applied independent from hospital volume in the participating centers $[4 \cdot \bullet, 12 \bullet]$. The current state of the art in Europe is the use of vagal nerve electrodes.

\section{Current Methods and Application of Continuous Nerve Monitoring}

\section{Vagal Nerve/RLN Based cIONM-Conventional cIONM}

As an initial step to any thyroid operation, the placement of the vagal nerve electrode requires dissection between the common carotid artery and the internal jugular vein for approximately 4-5 mm (Fig. 1A). For vagal nerve/RLN stimulation based cIONM, following the placement of a vagal nerve electrode, the vagal motor fibers are exposed to repetitive stimuli (1-2 mA, 0.1-0.2 ms duration, 0.6-3 Hz) [9••]. Analogously to the intermittent IONM setup, compound muscle action potentials are registered at the vocal fold level of the larynx using endotracheal tube surface electrodes (Fig. 1A) [29-31]. For the baseline, at least $500 \mu \mathrm{V}$ should be measured. If the baseline is lower, the tube's position should be optimized [4・•, $9 \cdot \bullet]$. For vagal nerve/RLN stimulation based cIONM, the simultaneous decrease of the EMG amplitude and the increase in its latency (combined event) indicate imminent impairment of RLN function or LOS, respectively [4••, 27, 39]. The INMSG recommended that a "combined event" with a decrease of the baseline amplitude of $>50 \%$, and an increase of the baseline latency of $>10 \%$ should prompt a change in the surgical maneuver, since a persistence of this signal constellation for $\geq 40 \mathrm{~s}$ was shown to be associated with an increased risk of postoperative VCP [4••]. Of interest is the reversibility of this combined event following changes in the surgical maneuver, e.g., pausing the operation [27, 39]. According to a recent study, after LOS (type 1 or 2), the recuperation of the RLN baseline amplitude to $\geq 50 \%$ of the initial signal can be considered a "complete recovery" [9••]. An intact postoperative vocal cord function can be expected in these cases. Therefore, contralateral lobectomy in the same sessioninstead of a two-staged thyroidectomy-is justified [9••]. Finally, it is recommended to document intraoperative EMG signals according to the algorithm of pre- and postoperative intermittent IONM assessment (V1-R1(-S1)-V2-R2(-S2)) [4••]. Important to mention is that the V2 signal should be elicited cranial (proximal) to the localization of the vagal nerve electrode, to assess potential nerve injury resulting from electrode positioning and to verify the intact function of the complete vagal and recurrent laryngeal nerve section within the operation field.

\section{Experimental LAR-cIONM as a Promising Novel Approach for cIONM}

An alternative method of performing continuous IONM takes advantage of the laryngeal adductor reflex, which can be triggered by supraglottic laryngeal mucosal stimulation (e.g., using $8 \mathrm{~mA}$ and $0.1-1 \mathrm{~ms}$ duration) [35•, 36, 37]. Afferent neural impulses are carried by the internal superior laryngeal nerve (IBSLN) to the brainstem. After a brainstem synapse, efferent impulses are carried via the vagal nerve and RLN to the larynx, resulting in bilateral vocal fold adduction that can be recorded via endotrachal tube surface electrodes (Fig. 1B) $[35 \cdot, 36]$. The resulting EMG signals are significantly lower when compared to conventional cIONM (Musholt et al., unpublished results). For a reliable baseline curve in LARcIONM, $>200 \mu \mathrm{V}$ should be measured [35•]. The existing threshold values for impeding LOS gained from analyses based on vagal nerve/RLN based cIONM currently require a verification in the field of LAR-cIONM. In 2018, Sinclair et al. described in a cohort of 134 nerves at risk that a LAR response decrease of $>50 \%$ of the initial baseline values indicated impeding nerve injury and that in case of an irreversible decrease $>60 \%$ of the initial baseline values a postoperative VCP can be expected [35 ${ }^{\circ}$. Moreover, reflex hyperexcitability was proposed as a potential indicator of permanent VCP, which, however, needs further validation in large patient cohorts [35•]. Due to the practical setup of cIONM signal stimulation and recording via the endotracheal tube, the technique appears particularly suitable for endoscopic approaches for thyroid surgery, since the additional placement of a vagal nerve electrode is not necessary.

\section{Anesthesia During IONM}

Cooperation with the anesthesiologists involved is essential to successful vagal nerve/RLN based intraoperative nerve monitoring in thyroid surgery [25]. Intubation using videolaryngoscopy allows for an optimal electrode positioning at the vocal fold level for signal recording (LION study, M. Kriege et al., unpublished results). Due to improvements of surface electrodes which includes an enlargement of the electrode length and an increase in the number from 2 to 4 electrodes with a dynamic change of the recording electrode, 
problems of electrode displacement during the operation were significantly reduced. Still, adequate fixation of the tube is important, as post-intubation patient positioning may lead to changes in the endotracheal tube electrode localization. Shortacting muscle relaxants should be avoided after the intubation period, to allow for valid IONM results [25]. For conventional vagal nerve/RLN based IONM, anesthesiologists have the freedom to choose the most appropriate anesthetic for the patient [25]. Typical anesthesia protocols would involve initial inhalation agents, as e.g., isoflurane or desflurane with or without nitrous oxide [25]. Also, a total intravenous anesthesia technique may be used for conventional vagal nerve/RLN based cIONM [25].

Similarly, for successful LAR-cIONM, direct contact of the stimulation/recording electrodes with the left and right laryngeal mucosa is crucial, which is why videolaryngoscopic intubation was recommended [35•]. If succinylcholine or other short-acting muscle relaxants are administered for intubation, 10 to 20 minutes should pass before obtaining a baseline in LAR-cIONM [35•]. For LAR-cIONM, total intravenous anesthesia currently presents the standard, since inhalational agents may abolish the cR1 response [35•]. A typical anesthesia can be induced with propofol and succinylcholine and maintained using total intravenous anesthesia with propofol and opioids (remifentanil) [35•].

\section{Controversies and Pitfalls}

\section{Which Patients Benefit from clONM?}

Numerous authors recommend cIONM for high-risk operations, e.g., thyroid cancer, redo surgery, Graves' disease, and large goiter. However, as described above, the predominant mechanism of nerve damage is related to traction injuries. In most cases, these are the result of the patient-specific anatomic situation at Berry's ligament (Fig. 2). Alterations of the RLN course by crossing vessels, which complicate preparation of the cricopharyngeal angle, cannot be foreseen. Consequently, it is not possible to predict before an operation, which patients may benefit from the use of cIONM. Moreover, the practical application of cIONM requires constant training and learning from the method itself [26]. In this sense, the immediate feedback from cIONM allows the surgeon to detect technical errors that might otherwise remain undetected. This goal cannot be achieved by a sporadic use of the method. On the contrary, the rare use of the technique may unsettle surgeons during more challenging procedures.

\section{Pitfalls}

Generally, the operating surgeon has to take into account potential equipment malfunction at any time during the operation. The so-called "laryngeal twitch," which describes the palpable contraction of the vocal fold muscles upon the stimulation of the vagal nerve, illustrates intact RLN function in absence of EMG signals and may uncover recording malfunction [8]. For both vagal nerve/RLN based and LAR-cIONM, the correct positioning of the endotracheal tube plays a key role. Therefore, the choice of the correct tube size is important, to facilitate an ideal contact between tube electrodes and larynx mucosa. In case of vagal nerve/ RLN based cIONM, impedance between tube electrode and larynx mucosa was recommended to be $<5 \mathrm{k} \Omega$ with a difference between the different sides of $<1 \mathrm{k} \Omega$ [8]. Moreover, for vagal nerve/RLN based IONM, optimal baseline amplitude values should initially exceed 500 $\mu \mathrm{V}$; otherwise, a correction of the endotracheal tube (and thereby the surface electrodes at the vocal fold level) by rotation or shifting should be attempted. Even during the operation, secondary tube dislocation has to be taken into consideration, when a decrease of the baseline amplitude (with unaffected signal latency) is observed. In case of LOS, a standard trouble shooting algorithm should be followed to differentiate technical errors from true LOS. After verification of the correct function of the technical equipment and exclusion of neuromuscular blockage, laryngeal twitch and/or the stimulation of the contralateral vagal nerve should be considered first. Positive EMG signals from the contralateral vagal nerve or circumscribed signal reduction along the ipsilateral RLN verify a true LOS. Negative or insufficient EMG signals from the contralateral vagal nerve may indicate displacement of the tube electrodes or neuromuscular blockage. During vagal nerve/RLN based cIONM the surgeon must be aware, that fluid collection around the vagal nerve electrode may impair the electrical current to the nerve and result in failure of stimulation. In case of the anatomic variant of a "nonrecurrent" RLN, positioning of the vagal electrode in a particularly cranial localization (before the separation point of the RLN) is necessary.

For LAR-cIONM, in case of an initial baseline of $<150$ $\mu \mathrm{V}$, the information cannot be considered reliable [35 ${ }^{\circ}$. Analogously, a change of the endotracheal tube position should be attempted, to improve baseline signals to values $>500 \mu \mathrm{V}$. In LAR-cIONM, stimulation of the supraglottic mucosa leads to bilateral activation of the laryngeal adductor reflex. So far, bilateral responses cannot simultaneously be registered, due to the current endotracheal tube/electrode design. Changes in the tube design in the future may allow for the simultaneous assessment of ipsiand contralateral LAR in order to understand, whether signal changes are caused by tube displacement, and the importance of far-field effects might be studied [35•]. Since 

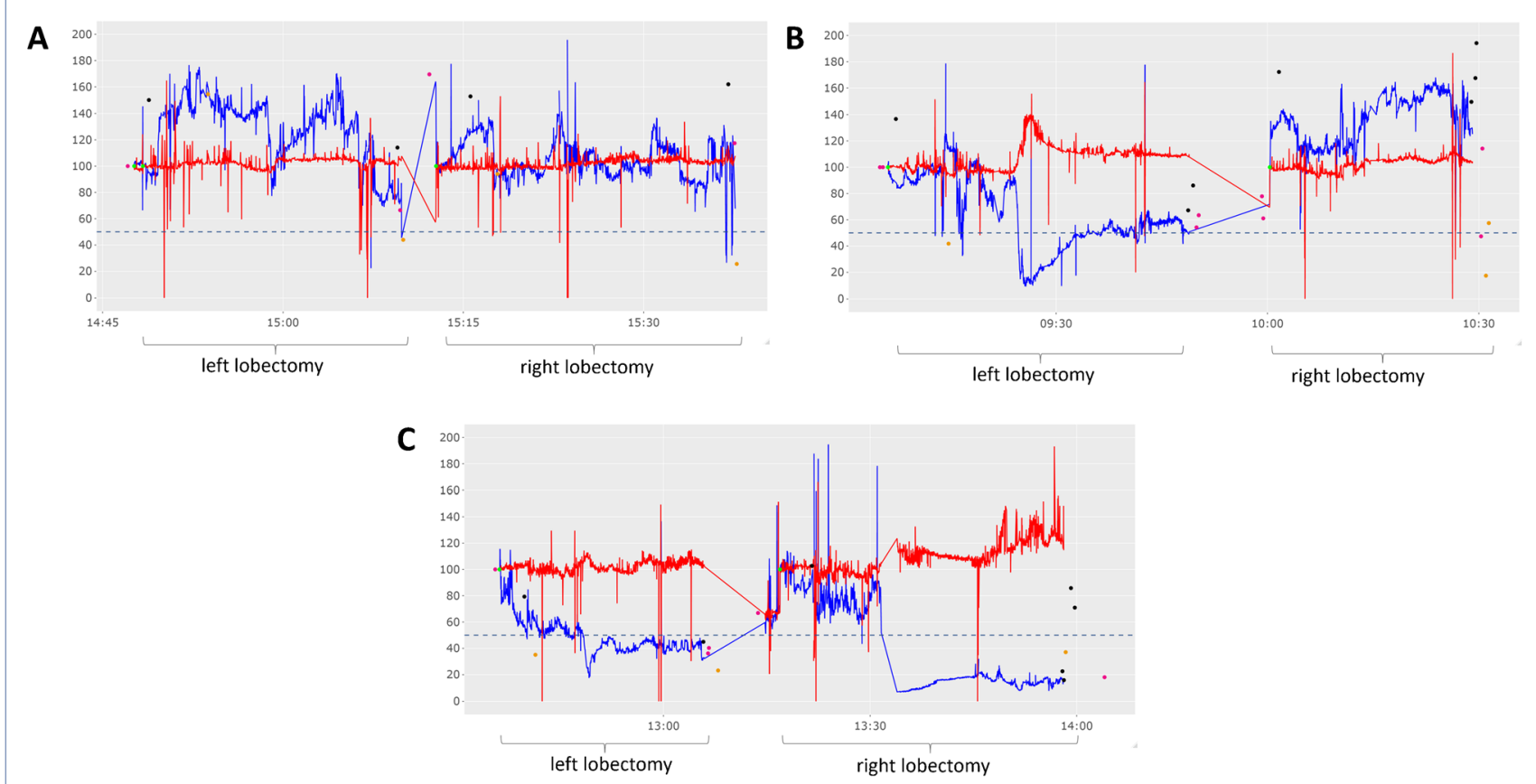

Fig. 2 Examples of recorded vagal/recurrent laryngeal nerve based cIONM signals during three different thyroidectomies (A-C). The Xaxis indicates the time of registration (hours to minutes). The Y-axis illustrates the percentage of amplitude $(\mathrm{mV})$ and latency (ms) with in relation to the chosen baseline, and the $50 \%$ threshold is marked with a dashed line. Reference points for the individually chosen baselines are coded as light green dots, which serve as a basis for the relative signal indication. Artefacts (immediately reversible decrease of the amplitude) can be distinguished from serious neural stress (dissociation of amplitude and latency with slow recovery). Waiting periods after signal reduction (intentionally paused preparation) are characterized by a reduction of movement artefacts. Results from intermittent stimulation of the vagal nerve ("V1" and "V2") are included in the graphs as pink dots, stimulation of the recurrent laryngeal nerve ("R1" and "R2") as black dots, and stimulation of the superior laryngeal nerve ("S1" and "S2") as orange dots. Light green dots mark the baseline setting. A Signal registration during thyroidectomy without significant changes during cIONM. The postoperative laryngoscopy verified normal vocal cord function. B Thyroidectomy with a drop of cIONM signal amplitude below the 50\% threshold during left lobectomy and signal recovery due to pausing of the resection. EMG signals at the end of initial left lobectomy exceeded $80 \%$ of the baseline $(>1 \mathrm{mV})$. The resection of the right lobe was consecutively performed without adverse events. Postoperative laryngoscopy remained without signs of vocal cord palsy. C Thyroidectomy with unambiguous drop of cIONM signal amplitude below the $50 \%$ threshold during resection of the right lobe, without sufficient signal recovery despite pausing the resection due to segmental, traction related injury (V2, $0.26 \mathrm{mV}$ after resection). Intermittent RLN stimulation at the end of the procedure verified reduced amplitudes at proximal stimulation and normal signal at distal stimulation, illustrating the right-sided segmental lesion. (The resection was continued after a marginal drop of the cIONM signal amplitude below the $50 \%$ threshold after initial left lobectomy since the absolute signal was $>1 \mathrm{mV}$, indicating a sufficient response.) A right-sided transient vocal cord palsy was registered by postoperative laryngoscopy
LAR-cIONM is a novel method, it was recommended to limit the method to centers with experienced neurophysiologists who support the operating surgeon to ensure that optimal LAR responses are obtained [35•].

\section{Conclusions}

IONM is a useful tool to avoid postoperative (bilateral) VCP after thyroid surgery. Whereas intermittent IONM allows for RLN "mapping” during tissue dissection, assessment of RLN lesion point/type of LOS, and decision guidance towards two-staged thyroidectomy, continuous IONM facilitates the real-time evaluation of the effect of a surgical maneuver on RLN function. Consequently, a more powerful effect on the reduction of postoperative transient/permanent $\mathrm{VCP}$ rate can be expected when using this "interactive" evolvement of the merely observatory technique of intermittent IONM. There are currently two main approaches for the performance of cIONM: conventional vagal nerve/ RLN based cIONM and experimental methods, which do not require the positioning of a vagal nerve electrode for signal stimulation. One of these methods is the recently described technique of LAR-cIONM that utilizes the laryngeal adductor reflex. For conventional vagal 
nerve/RLN based cIONM, comprehensive analyses of threshold values and impact of signal changes on postoperative VCP rates were reported. For the experimental techniques, e.g., LAR-cIONM, initial results are available, but further investigation is required.

Funding Open Access funding enabled and organized by Projekt DEAL.

\section{Declarations}

Conflict of Interest Dr. Staubitz has nothing to disclose. Prof. Dr. Musholt has received project-related research funds and honoraria from INOMED, IPSEN, Novartis, Sanfi/Genzyme/Henning, Shire, and Takeda.

Human and Animal Rights and Informed Consent All reported studies/ experiments with human or animal subjects performed by the authors have been previously published and complied with all applicable ethical standards (including the Helsinki declaration and its amendments, institutional/national research committee standards, and international/national/institutional guidelines).

Open Access This article is licensed under a Creative Commons Attribution 4.0 International License, which permits use, sharing, adaptation, distribution and reproduction in any medium or format, as long as you give appropriate credit to the original author(s) and the source, provide a link to the Creative Commons licence, and indicate if changes were made. The images or other third party material in this article are included in the article's Creative Commons licence, unless indicated otherwise in a credit line to the material. If material is not included in the article's Creative Commons licence and your intended use is not permitted by statutory regulation or exceeds the permitted use, you will need to obtain permission directly from the copyright holder. To view a copy of this licence, visit http://creativecommons.org/licenses/by/4.0/.

\section{References}

Papers of particular interest, published recently, have been highlighted as:

- Of importance

•- Of major importance

1. Gardner GM, Smith MM, Yaremchuk KL, Peterson EL. The cost of vocal fold paralysis after thyroidectomy. The Laryngoscope. 2013;123(6):1455-63. https://doi.org/10.1002/lary.23548.

2. Wu CW, Dionigi G, Barczynski M, Chiang FY, Dralle H, Schneider R, et al. International neuromonitoring study group guidelines 2018: part II: optimal recurrent laryngeal nerve management for invasive thyroid cancer-incorporation of surgical, laryngeal, and neural electrophysiologic data. The Laryngoscope. 2018;128(Suppl 3):S18-s27. https://doi.org/10.1002/lary.27360.

3. Nouraei SAR, Allen J, Kaddour H, Middleton SE, Aylin P, Darzi A, et al. Vocal palsy increases the risk of lower respiratory tract infection in low-risk, low-morbidity patients undergoing thyroidectomy for benign disease: a big data analysis. Clin Otolaryngol. 2017;42(6):1259-66. https://doi.org/10.1111/coa.12913.
4.• Schneider R, Randolph GW, Dionigi G, Wu CW, Barczynski M, Chiang FY, et al. International neural monitoring study group guideline 2018 part I: staging bilateral thyroid surgery with monitoring loss of signal. Laryngoscope. 2018;128(Suppl 3):S1-S17. https://doi.org/10.1002/lary.27359 The publication outlines the basic principles of intraoperative nerve monitoring and current state-of-the-art International Neural Monitoring Study Group (INMSG) guidelines.

5. Schneider R, Sekulla C, Machens A, Lorenz K, Thanh PN, Dralle $\mathrm{H}$. Dynamics of loss and recovery of the nerve monitoring signal during thyroidectomy predict early postoperative vocal fold function. Head Neck. 2016;38(Suppl 1):E1144-51. https://doi.org/10. 1002/hed.24175.

6. Schneider R, Randolph G, Dionigi G, Barczynski M, Chiang FY, Triponez F, et al. Prospective study of vocal fold function after loss of the neuromonitoring signal in thyroid surgery: the International Neural Monitoring Study Group's POLT study. The Laryngoscope. 2016;126(5):1260-6. https://doi.org/10.1002/lary.25807.

7. Chiang FY, Lu IC, Kuo WR, Lee KW, Chang NC, Wu CW. The mechanism of recurrent laryngeal nerve injury during thyroid surgery-the application of intraoperative neuromonitoring. Surgery. 2008;143(6):743-9. https://doi.org/10.1016/j.surg.2008. 02.006 .

8. Dralle H, Lorenz K, Schabram P, Musholt TJ, Dotzenrath C, Goretzki PE, et al. Intraoperative neuromonitoring in thyroid surgery. Recommendations of the Surgical Working Group for Endocrinology. Chirurg. 2013;84(12):1049-56. https://doi.org/10. 1007/s00104-013-2656-z.

9.• Schneider R, Randolph G, Dionigi G, Barczynski M, Chiang FY, $\mathrm{Wu} \mathrm{CW}$, et al. Prediction of postoperative vocal fold function after intraoperative recovery of loss of signal. Laryngoscope. 2019;129(2):525-31. https://doi.org/10.1002/lary.27327 This multicenter study determined an important threshold of amplitude recovery (50 per cent of initial baseline) for normal early postoperative vocal fold function after segmental type 1 and after global type 2 loss of signal in continuous intraoperative nerve monitoring.

10. McManus C, Luo J, Sippel R, Chen H. Is thyroidectomy in patients with Hashimoto thyroiditis more risky? J Surg Res. 2012;178(2): 529-32. https://doi.org/10.1016/j.jss.2012.09.017.

11. Kwon H, Kim JK, Lim W, Moon BI, Paik NS. Increased risk of postoperative complications after total thyroidectomy with Graves' disease. Head Neck. 2019;41(2):281-5. https://doi.org/10.1002/ hed.25484.

12. Staubitz JI, Watzka F, Poplawski A, Riss P, Clerici T, Bergenfelz A, et al. Effect of intraoperative nerve monitoring on postoperative vocal cord palsy rates after thyroidectomy: European multicentre registry-based study. BJS Open. 2020;4(5):821-9. https://doi.org/ $10.1002 /$ bjs 5.50310 This multicenter study demonstrated that currently intraoperative nerve monitoring is widely used in European centers of endocrine surgery and that the use of the technique was associated with a significantly reduced postoperative vocal cord palsy rate.

13. Bergenfelz A, Salem AF, Jacobsson H, Nordenstrom E, Almquist $\mathrm{M}$. Risk of recurrent laryngeal nerve palsy in patients undergoing thyroidectomy with and without intraoperative nerve monitoring. Br J Surg. 2016;103(13):1828-38. https://doi.org/10.1002/bjs. 10276.

14. Dralle H, Sekulla C, Haerting J, Timmermann W, Neumann HJ, Kruse E, et al. Risk factors of paralysis and functional outcome after recurrent laryngeal nerve monitoring in thyroid surgery. Surgery. 2004;136(6):1310-22. https://doi.org/10.1016/j.surg.2004.07.018.

15. Godballe C, Madsen AR, Sorensen CH, Schytte S, Trolle W, Helweg-Larsen J, et al. Risk factors for recurrent nerve palsy after thyroid surgery: a national study of patients treated at Danish departments of ENT Head and Neck Surgery. Eur Arch 
Otorhinolaryngol. 2014;271(8):2267-76. https://doi.org/10.1007/ s00405-013-2767-7.

16. Thomusch O, Sekulla C, Walls G, Machens A, Dralle H. Intraoperative neuromonitoring of surgery for benign goiter. Am J Surg. 2002;183(6):673-8. https://doi.org/10.1016/s00029610(02)00856-5.

17. Musholt TJ, Bockisch A, Clerici T, Dotzenrath C, Dralle H, Goretzki PE, et al. Update of the S2k guidelines : surgical treatment of benign thyroid diseases. Chirurg. 2018;89(9):699-709. https:// doi.org/10.1007/s00104-018-0653-y.

18. Dralle H, Musholt TJ, Schabram J, Steinmuller T, Frilling A, Simon D, et al. German Association of Endocrine Surgeons practice guideline for the surgical management of malignant thyroid tumors. Langenbecks Arch Surg. 2013;398(3):347-75. https://doi.org/10. 1007/s00423-013-1057-6.

19. Higgins TS, Gupta R, Ketcham AS, Sataloff RT, Wadsworth JT, Sinacori JT. Recurrent laryngeal nerve monitoring versus identification alone on post-thyroidectomy true vocal fold palsy: a metaanalysis. The Laryngoscope. 2011;121(5):1009-17. https://doi.org/ 10.1002/lary.21578.

20. Pisanu A, Porceddu G, Podda M, Cois A, Uccheddu A. Systematic review with meta-analysis of studies comparing intraoperative neuromonitoring of recurrent laryngeal nerves versus visualization alone during thyroidectomy. J Surg Res. 2014;188(1):152-61. https://doi.org/10.1016/j.jss.2013.12.022.

21. Zheng S, Xu Z, Wei Y, Zeng M, He J. Effect of intraoperative neuromonitoring on recurrent laryngeal nerve palsy rates after thyroid surgery-a meta-analysis. Journal of the Formosan Medical Association = Taiwan yi zhi. 2013;112(8):463-72. https://doi.org/ 10.1016/j.jfma.2012.03.003

22. Jatzko GR, Lisborg PH, Muller MG, Wette VM. Recurrent nerve palsy after thyroid operations-principal nerve identification and a literature review. Surgery. 1994;115(2):139-44.

23. Calò PG, Pisano G, Medas F, Pittau MR, Gordini L, Demontis R, et al. Identification alone versus intraoperative neuromonitoring of the recurrent laryngeal nerve during thyroid surgery: experience of 2034 consecutive patients. Journal of otolaryngology - head \& neck surgery $=$ Le Journal d'oto-rhino-laryngologie et de chirurgie cervico-faciale. 2014;43(1):16. https://doi.org/10.1186/1916-021643-16.

24. Hermann M, Hellebart C, Freissmuth M. Neuromonitoring in thyroid surgery: prospective evaluation of intraoperative electrophysiological responses for the prediction of recurrent laryngeal nerve injury. Annals of surgery. 2004;240(1):9-17. https://doi.org/10. 1097/01.sla.0000132260.34503.02.

25. Randolph GW, Dralle H, Abdullah H, Barczynski M, Bellantone R, Brauckhoff $\mathrm{M}$, et al. Electrophysiologic recurrent laryngeal nerve monitoring during thyroid and parathyroid surgery: international standards guideline statement. The Laryngoscope. 2011;121(Suppl 1):S1-16. https://doi.org/10.1002/lary.21119.

26. Schneider R, Machens A, Randolph GW, Kamani D, Lorenz K, Dralle H. Opportunities and challenges of intermittent and continuous intraoperative neural monitoring in thyroid surgery. Gland Surg. 2017;6(5):537-45. https://doi.org/10.21037/gs.2017.06.08.

27. Phelan E, Schneider R, Lorenz K, Dralle H, Kamani D, Potenza A, et al. Continuous vagal IONM prevents recurrent laryngeal nerve paralysis by revealing initial EMG changes of impending neuropraxic injury: a prospective, multicenter study. The Laryngoscope. 2014;124(6):1498-505. https://doi.org/10.1002/ lary. 24550
28. Angeletti F, Musholt PB, Musholt TJ. Continuous intraoperative neuromonitoring in thyroid surgery. Surgical technology international. 2015;27:79-85.

29. Schneider R, Bures C, Lorenz K, Dralle H, Freissmuth M, Hermann $\mathrm{M}$. Evolution of nerve injury with unexpected EMG signal recovery in thyroid surgery using continuous intraoperative neuromonitoring. World journal of surgery. 2013;37(2):364-8. https://doi.org/10.1007/s00268-012-1853-0.

30. Schneider R, Przybyl J, Pliquett U, Hermann M, Wehner M, Pietsch UC, et al. A new vagal anchor electrode for real-time monitoring of the recurrent laryngeal nerve. Am J Surg. 2010;199(4):507-14. https://doi.org/10.1016/j.amjsurg.2009.04.036.

31. Ulmer C, Koch KP, Seimer A, Molnar V, Meyding-Lamadé U, Thon KP, et al. Real-time monitoring of the recurrent laryngeal nerve: an observational clinical trial. Surgery. 2008;143(3):359 65. https://doi.org/10.1016/j.surg.2007.10.007.

32. Suh I, Yingling C, Randolph GW, Duh QY. A novel method of neuromonitoring in thyroidectomy and parathyroidectomy using transcutaneous intraoperative vagal stimulation. JAMA Surg. 2016;151(3):290-2. https://doi.org/10.1001/jamasurg.2015.3249.

33. Zhao Y, Li C, Zhang D, Li S, Wang T, Dionigi G, et al. Continuous neural monitoring in endoscopic thyroidectomy: feasibility experimental study for transcutaneous vagal nerve stimulation. J Laparoendosc Adv Surg Tech A. 2020;30(10):1095-101. https:// doi.org/10.1089/lap.2020.0073.

34. Ichino T, Tanaka S, Tanaka R, Tanaka N, Ishida T, Sugiyama Y, et al. Transcranial motor-evoked potentials of laryngeal muscles for intraoperative neuromonitoring of the vagus nerve during thyroid surgery. Journal of Anesthesia. 2019;33(2):221-9. https://doi.org/ 10.1007/s00540-018-2601-x.

35. Sinclair CF, Tellez MJ, Ulkatan S. Noninvasive, tube-based, continuous vagal nerve monitoring using the laryngeal adductor reflex: feasibility study of 134 nerves at risk. Head Neck. 2018;40(11):2498-506. https://doi.org/10.1002/hed.25377 In this publication, the methodology of noninvasive laryngeal adductor reflex-based continuous IONM (LAR-C-IONM) was introduced and validated in $\mathbf{1 0 0}$ patients.

36. Sinclair CF, Téllez MJ, Ulkatan S. Continuous laryngeal adductor reflex versus intermittent nerve monitoring in neck endocrine surgery. The Laryngoscope. 2020;131:230-6. https://doi.org/10.1002/ lary. 28710 .

37. Sinclair CF, Téllez MJ, Tapia OR, Ulkatan S, Deletis V. A novel methodology for assessing laryngeal and vagus nerve integrity in patients under general anesthesia. Clin Neurophysiol. 2017;128(7): 1399-405. https://doi.org/10.1016/j.clinph.2017.03.002.

38. Barczynski M, Konturek A, Cichon S. Randomized clinical trial of visualization versus neuromonitoring of recurrent laryngeal nerves during thyroidectomy. Br J Surg. 2009;96(3):240-6. https://doi.org/ $10.1002 /$ bjs. 6417 .

39. Schneider R, Randolph GW, Sekulla C, Phelan E, Thanh PN, Bucher M, et al. Continuous intraoperative vagus nerve stimulation for identification of imminent recurrent laryngeal nerve injury. Head \& neck. 2013;35(11):1591-8. https://doi.org/10.1002/hed. 23187.

Publisher's Note Springer Nature remains neutral with regard to jurisdictional claims in published maps and institutional affiliations. 